\title{
Review of Clinical Trials in COVID-19: Trying to Solve the Clinicians' Conundrum
}

\author{
Amita Athavale ${ }^{1}$ Vijay Khatri ${ }^{1} \quad$ Tanya Athavale ${ }^{1}$ \\ ${ }^{1}$ Department of Pulmonary Medicine and Environmental \\ Pollution Research Centre, Seth GS Medical College and \\ KEM Hospital, Mumbai, India \\ 2Department of Radiology, Seth GS Medical College and \\ KEM Hospital, Mumbai, India \\ ${ }^{3}$ Department of Microbiology, Seth GS Medical College and \\ KEM Hospital, Mumbai, India \\ ${ }^{4}$ Department of Pulmonary Medicine, Seth GS Medical College and \\ KEM Hospital, Mumbai, India
}

\author{
Hemant Deshmukh² Gita Nataraj ${ }^{3} \quad$ Rahul Bahot $^{4}$
}

Ann Natl Acad Med Sci (India):2020;3:119-125

\author{
Address for correspondence Amita Athavale, MD (TB \& Chest \\ Medicine), Department of Pulmonary Medicine and Environmental \\ Pollution Research Centre, Seth GS Medical College and KEM \\ Hospital, Mumbai 400012, India \\ (e-mail: amitaathavale@rediffmail.com).
}

\begin{abstract}
Keywords

- COVID-19

- Remdesivir

- Favipiravir

- Lopinavir/Ritonavir

- immunomodulators

- convalescent plasma

SARS-CoV-2 and the resulting "COVID-19" has escalated into a pandemic and has resulted in significant morbidity and mortality. Currently, there is no approved antiviral treatment available. Few antiviral agents are undergoing clinical trials for their safety and efficacy against COVID-19. With various repurposed drugs under evaluation, treatment approaches range from search for an effective antiviral, immunomodulation and anticoagulation, use of point-of-care ultrasound to novel ventilatory strategies. Newer agents targeting the inflammatory pathway are also under investigation. There are more than 1955 ongoing clinical trials of repurposed and novel agents. Here we review some of the ongoing trials that have published, at least, interim results of treating patients with COVID-19.
\end{abstract}

\section{Introduction}

SARS-COV-2 and the resulting "COVID-19" has escalated into a pandemic and threatens the existence of humanity today like no other modern-day disaster. While COVID-19 has plunged countries and economies into a struggle for survival, it also poses a great challenge to clinicians, who are trying to treat and cure patients while protecting themselves and their colleagues at the same time.

In the day of evidence-based and personalized medicine, it is a clinicians' conundrum to analyze and make sense of the enormous data available today. The sheer numbers of COVID-19 patients along with the fact that the world is still in search of a magic pill, which acts against this disease, has led to numerous clinical trials and an even more number of drugs and strategies. Some of these are promising and some just speculative.

For a clinician, statistically significant results do matter. However, even major is the patient being treated, who may constitute the tiniest fraction of the giant "big data." To a clinician, that patient is the whole $100 \%$. This article aims to review ongoing clinical trials and published data, particularly focusing on the therapeutic aspects to examine what has worked for us so far, in what type of patients and in which combinations, as we spar with COVID-19.

\section{Virology}

A member of the family of Coronaviridae viruses, SARS-CoV-2, an enveloped virus possesses a positive-sense, single-stranded RNA genome. The family is characterized by significant genetic variability and high-recombination rates. Some of these are innocuous and do not cause life-threatening disease. Some of the variants, however, cause pandemics-Severe Acute Respiratory Syndrome (SARS-CoV) in 2003, Middle East respiratory syndrome coronavirus (MERS-CoV) in 2012 and, now, 2019 novel coronavirus (SARS-CoV-2) in 2019-2020. ${ }^{1}$

SARS-CoV-2 is a relatively large virus at 60 to $100 \mathrm{~nm}$ and is spherical in shape. Over a period of time, two major 
subtypes have been identified-a L variant, found to be more aggressive and contagious and a $\mathrm{S}$ variant, presumed to be less aggressive and contagious. ${ }^{1}$

Transmission of SARS-CoV-2 has been predominantly found to be via respiratory droplets (> 5-10 um diameter) and contact routes. However, recent evidence suggests airborne transmission via respiratory droplet nuclei at least in certain clinical situations, WHO recommends airborne precautions for circumstances and settings in which aerosol-generating procedures and support treatment are performed, according to risk assessment. ${ }^{2}$

\section{Epidemiology}

As of May 7, 2020, according to the COVID-19 tracker, there are 35902 active cases of COVID-19 in India. We have lost 1783 patients and 15266 patients have been cured or discharged. ${ }^{3}$

Epidemiological studies the world over have estimated the incubation period of COVID-19 to be approximately 4 to 6 days. The proportion of cases taking at least 14 days to manifest symptoms has been pegged at 1 to $5 \%$ in varying studies.,

\section{Clinical Presentation}

Although fever is the most consistent symptom in COVID-19, seen in $85 \%$ of cases at least some time during the disease course, only $45 \%$ patients have been found to be febrile on early presentation. Cough has been seen in $67.7 \%$ of patients and this is associated with sputum production in $33.4 \%$ cases. Other respiratory symptoms such as dyspnea, sore throat, and nasal congestion present in $18.6 \%, 13.9 \%$, and $4.8 \%$ of cases, respectively. Body aches, chills, and headache are seen in $14.8 \%, 11.4 \%$ and $13.6 \%$ of the cases, respectively. ${ }^{6}$

Several case series report gastrointestinal symptoms, with percentages varying between 2 to $40 \%$ of patients. Dysgeusia and anosmia were noted in up to $53 \%$ of the cases in a small cohort from Italy. ${ }^{7}$

Neurological symptoms ranging from dizziness and headache to ischemic or hemorrhagic stroke have been reported in case series from China and the US. Some cases of Guillain-Barré syndrome and acute necrotizing encephalopathy have also been reported. ${ }^{7}$

COVID-19 has been found to be associated with a hypercoagulable state, an increased risk of venous thromboembolism, and pulmonary embolism in a retrospective cohort study in China. There are various cardiovascular events that have been associated with COVID-19 and include myocarditis, pericarditis and myocardial injury, resulting in reduced systolic function, cardiac arrhythmias, heart failure, and these have at times mimicked acute coronary syndromes. ${ }^{7}$

With SARS-CoV-2 RNA detected in tears of infected patients in a Chinese case series, the virus does not spare even eyes-ocular manifestations including chemosis were reported in approximately $32 \%$ patients. $^{7}$

In the Indian context, respiratory syndromes in COVID-19 have been classified based on their clinical severity. ${ }^{8}$
These include the following:

1. Uncomplicated illness: nonspecific symptoms such as fever, sore throat, nasal congestion, malaise, headache.

2. Acute respiratory distress syndrome.

3. Sepsis.

4. Septic shock.

5. Mild pneumonia: patients with pneumonia without any signs of severe pneumonia.

6. Severe pneumonia.

\section{Unmet Medical Need}

As of May 7, 2020, there are 3672270 confirmed cases, 254045 deaths due to COVID-19 and 215 countries affected by COVID-19 in the world.

Although the entire world is trying its best to find a cure for COVID-19, after more than 4 months of the first case of COVID-19 in the world, there is no treatment with confirmed efficacy yet. According to WHO, there are 1955 COVID-19 clinical trials currently registered on the International Clinical Trials Registry platform.

\section{Treatments Evaluated for COVID-19 Clinical Trials}

Therapies for COVID-19 may be classified as per their targets. The first set of therapies act on SARS-CoV-2 directly, either by inhibiting crucial viral enzymes responsible for genome replication or blocking viral entry to human cells. The second set is that of drugs that have potential immunomodulatory effects on the human immune system. This may be in the form of boosting the immune system, particularly the innate immune response that primarily acts against viruses or in the form of toning down the immune response, thereby inhibiting the cascade of inflammation that leads to lung injury.

It is important to note that the drugs currently being evaluated and consequently used for COVID-19 are mainly repurposed drugs and await phase III clinical trials for the treatment of this 4-month-old disease.

\section{Remdesivir}

Remdesivir is a monophos phosphoramidate prodrug of adenosine analog remdesivir-triphosphate (RDV-TP). RDV-TP inhibits RNA-dependent RNA polymerase (RdRps). RDV-TP competes with adenosine triphosphate for incorporation into nascent viral RNA chains, and once incorporated into viral RNA at position I, it terminates RNA synthesis at position I+3. Since RDV-TP causes chain termination 3 additional nucleotides later, it is thought to evade proofreading by viral exoribonuclease.

Patients with SARS-CoV-2 infection with time from symptom onset of less than 12 days, hypoxemia (oxygen saturation $\leq 94 \%$ at room air or $\mathrm{PaO} 2 / \mathrm{FiO} 2$ ratio of $\leq 300$ ), and a radiologically confirmed pneumonia were included in a randomized, double-blind, placebo-controlled multicentre trial by Wang et al. 
Patients fulfilling the inclusion criteria were randomly assigned in a 2:1 ration to receive IV remdesivir (200 $\mathrm{mg}$ on day 1 followed by $100 \mathrm{mg}$ on day 2 to day 10 ) or same volume of placebo infusions for 10 days. Concomitant use of corticosteroids, interferon, and lopinavir/ritonavir were allowed in this trial.

The primary endpoint in this trial was the time to clinical improvement up to day 28 from the day of randomization. Using remdesivir was not associated with a difference in time to clinical improvement (hazard ratio 1.23 [95\% CI $0 \cdot 87-1.75]$ ). Although not statistically significant, findings in this trial were suggestive of improved outcomes with early treatment, as patients receiving remdesivir had a numerically faster time to clinical improvement than those receiving placebo among patients with symptom duration of 10 days or less (hazard ratio 1.52 [0.95-2.43]). ${ }^{9}$

Adverse events were reported in 102 (66\%) of 155 remdesivir recipients versus 50 (64\%) of 78 placebo recipients. ${ }^{9}$ The 28-day mortality in the remdesivir group was numerically lower when it was used within 10 days of symptom onset. However, remdesivir group patients had numerically higher mortality with late use of the drug. There was no significant difference in the overall mortality in the two groups (22 [14\%] died in the remdesivir group vs. $10(13 \%)$ in the placebo group; difference $1 \cdot 1 \%$ [95\% CI-8.1 to $10 \cdot 3])^{9}$

Remdesivir was used in hypoxemic patients (oxygen saturation of $\leq 94 \%$ at room air or receiving oxygen support) on compassionate grounds by Grein et al. A 10-day course of remdesivir was planned with IV administration of $200 \mathrm{mg}$ on day 1 , followed by $100 \mathrm{mg}$ daily from day 2 to day $10 .{ }^{10}$ Of the 61 patients, data for 53 patients could be analyzed by the investigator (posttreatment data was not available for seven patients and one experienced a dosing error).

At the beginning of the study, 30 patients $(57 \%)$ were receiving mechanical ventilation and four (8\%) were receiving extracorporeal membrane oxygenation. As many as 17 out of 30 patients (57\%) were extubated, and 36 patients (68\%) had an improvement in oxygen support class during a median follow-up of 18 days.

A total of 25 patients (47\%) could be discharged. Mortality rate in the study was $13 \%$ (seven patients); $18 \%$ of patients receiving invasive ventilation ( 6 of 34 ) and one patient from among those not requiring mechanical ventilation died. ${ }^{10}$

Adverse events during follow-up were reported in a total of 32 patients (60\%). These were more common in patients who had received invasive mechanical ventilation. The most common adverse events were increase in hepatic enzymes, diarrhea, rash, renal impairment, and hypotension. Twelve patients had serious adverse events including multiple organ dysfunction syndrome (MODS), septic shock and acute kidney injury. ${ }^{10}$

Gilead, a pharmaceutical company, is currently conducting two randomized, open-label, multicentre, phase 3 clinical studies to evaluate the safety and efficacy of two dosing durations -5 days and 10 days-of remdesivir in adults diagnosed with COVID-19.
The investigators announced results of the SIMPLE 1 trial on April 29, 2020.

Patients included in the study had evidence of pneumonia and hypoxemia but did not require mechanical ventilation at the time of study entry.

As per the results revealed by the investigators, 50\% patients in the 5-day treatment group achieved clinical improvement in 10 days and required 11 days in the 10-day treatment group.

While more than half of patients in both treatment groups were discharged from the hospital by day 14 (5-day: $60.0 \%, n=120 / 200$ vs. 10 -day: $52.3 \% n=103 / 197 ; p=0.14$ ), 64.5 percent $(n=129 / 200)$ of patients in the 5 -day treatment group and 53.8 percent $(n=106 / 197)$ of patients in the 10-day treatment group achieved clinical recovery by this time. ${ }^{11}$

Published results from this study highlight better response with early treatment. Across treatment arms, by day 14, 62 percent of patients treated early(within 10 days of symptom onset) were able to be discharged from the hospital, compared with 49 percent of patients who were treated late (beyond 10 days of symptom onset). ${ }^{11}$

The most common adverse events reported in either group were nausea (5-day: $10.0 \%, n=20 / 200$ vs. 10 -day: $8.6 \%$, $n=17 / 197)$ and acute respiratory failure (5-day: 6.0\%, $n=12 / 200$ vs. 10 -day: $10.7 \%, n=21 / 197)$. Grade 3 or higher liver enzyme (ALT) elevations occurred in $7.3 \%(n=28 / 385)$ of patients and $3 \%(n=12 / 397)$ of patients who discontinued Remdesivir treatment due to elevated liver enzymes. ${ }^{11}$

\section{Favipiravir}

Favipiravir (FPV) is the prodrug of purine nucleotide favipiravir ribofuranosyl-5'- triphosphate that inhibits RNA polymerase on activation and halts viral replication.

An open-label, nonrandomized, before-after controlled study was conducted at Shenzen in China from January 30 to February 14, 2020. Patients included in this study were aged between 16 to 75 years, had nasopharyngeal swab samples tested positive for SARS-CoV-2 RNA, and had disease onset duration of less than 7 days. Patients with more severe disease, as evidenced by any organ system failure, were excluded from the study.

Eligible patients were included in the FPV arm of the study. Patients who had initially been treated with antiviral therapy with lopinavir/ritonavir (LPV/r) from January 24 to January 30, 2020, served as the control arm. ${ }^{12}$

The treatment regimen for FPV arm consisted of orally administered FPV at a dose of $1600 \mathrm{mg}$ twice daily on day 1 , followed by $600 \mathrm{mg}$ twice daily from days 2 to 10 .

Patients in the LPV/RTV arm were given oral dose of LPV $400 \mathrm{mg} / \mathrm{RTV} 100 \mathrm{mg}$ twice daily. Patients in both arms received IFN- $\alpha 1 \mathrm{~b} 60$ ug twice daily in aerosolized form. A background care regimen of supplemental oxygen, oral or intravenous hydration, electrolyte correction, antipyretics, analgesics and antiemetic drugs was instituted for both arms of the study..$^{12}$

Investigators observed a shorter viral clearance time for the FPV arm (median [interquartile range, IQR], FPV: 4 [2.5-9] d vs. control:11 [8-13] d, $p<0.001)$. FPV arm also showed 
significant improvement in chest imaging (FPV: $91.43 \%$ vs. control:62.22\% $[p=0.004])^{12}$

\section{Lopinavir/Ritonavir}

Lopinavir/ritonavir (LPV/r) are antiretroviral protease inhibitors, commonly used in second line antiretroviral therapy in our country. Preclinical data suggests that these drugs may have some efficacy against Coronaviruses.

In a report by Young and colleagues about the outcomes of the first 18 patients infected with SARS-CoV-2 in Singapore, the authors observed reduction in oxygen requirements of 3 out of 5 patients treated with LPV/r. Two patients progressed to respiratory failure. ${ }^{13}$

Cao and colleagues conducted an open-label randomized trial with 199 patients who were afflicted with COVID-19 pneumonia. ${ }^{14}$ The patients were randomized to treatment arm with LPV/r 400/100 mg administered twice daily $(n=99)$ and standard care $(n=100)$. No significant difference was observed in the time to clinical improvement (LPV/r: 16 [13-17] days vs. standard therapy:16 [15-17] days), time until discharge (LPV/r: 12 [10-16] days vs. standard therapy:14 [11-16] days), or mortality (LPV/r: $19.2 \%$ vs. standard therapy: $25.0 \%$; absolute difference-5.8, 95\% confidence interval-17.3 to $5.7 \%$ ) between the two study arms. There was no difference in reduction of viral loads over time between the two groups. ${ }^{14}$

Hung et al have published results of an open-label, randomized, phase 2 trial conducted at six centers in Hong Kong. ${ }^{15}$ In this trial, patients with SARS-CoV-2 infection with a time since symptom onset of less than 14 days were randomized in a 2:1 distribution to receive a 14-day therapy comprising lopinavir $400 \mathrm{mg}$ and ritonavir $100 \mathrm{mg}$ twice a day, along with ribavirin $400 \mathrm{mg}$ every 12 hours and three doses of 8 million IU units of interferon $\beta-1 \mathrm{~b}$ on alternate days. The control group received only $\mathrm{LPV} / \mathrm{r}$ combination. ${ }^{15}$

Of the 127 patients recruited, 86 patients received combination therapy. The group receiving combination therapy had a significantly shorter median time from start of treatment to negative nasopharyngeal swab compared with the control group ( 7 days [IQR 5-11] vs. 12 days [IQR 8-15]). While there were no differences in adverse events reported in the two groups, no patients died during the study. It is pertinent to note that this benefit was not demonstrated in late treatment. 15,16

There are published case reports from Korea and China comprising a total of six patients that describe decreased viral load and clinical improvement after LPV/r initiation. However, it is rather difficult to interpret these data due to heterogeneity and lack of comparator treatments. ${ }^{17}$

\section{Chloroquine and Hydroxychloroquine}

Chloroquine is an antimalarial drug that has been on investigators' radar for its potential use against SARS$\mathrm{CoV}-2$. It has been shown to inhibit viral protein glycosylation, virus assembly, new virus particle transport, and virus release. Other proposed mechanisms of action also involve ACE2 cellular receptor inhibition, inhibiting fusion of the virus by acidification at the surface of the cell membrane, and immunomodulation via cytokine pathway. Hydroxychloroquine (HCQ) has a similar mechanism of action.

Initial experience with HCQ published by Gautret and colleagues has been in discussion over the past few weeks. As many as 36 COVID-19 patients were divided into two groups. HCQ group had 20 patients and received hydroxychloroquine $200 \mathrm{mg}$ orally every 8 hours. There were 16 patients in the control group. ${ }^{18}$

The investigators observed that hydroxychloroquine $(14 / 20,70 \%)$ was superior to control $(2 / 16,12.5 \% ; p=0.001)$ in eradicating SARS-CoV-2 from the nasopharynx.

Of the 20 patients in HCQ group, six patients had received azithromycin to prevent bacterial super infection. Investigators demonstrated numerically superior viral eradication in the combination subgroup when compared with HCQ-only subgroup $([6 / 6,100 \%]$ vs. $(8 / 14,57 \%])$. Although authors in this study concluded that azithromycin reinforced action of HCQ in viral load reduction, it is pertinent to note that both these drugs cause QTc prolongation. ${ }^{18}$

\section{Ivermectin}

Ivermectin, an antiparasitic agent used commonly in our country, can dissociate the preformed IMP $\alpha / \beta 1$ heterodimer that is responsible for the nuclear transport of nuclear protein cargo. Investigators have demonstrated approximately 5000 -fold reduction in viral RNA at 48 hours with a single addition of Ivermectin to Vero-hSLAM cells 2 hours postinfection with SARS-CoV-2. ${ }^{19,20}$

\section{Anticoagulation}

COVID-19 has presented as a hypercoagulable state in some studies. Indeed, investigators have found evidence suggesting that the cause of severe hypoxemia in this disease could be microthrombi in the pulmonary circulation. There are several reports of patients presenting with symptoms resembling those of acute coronary syndrome.

Tang et al retrospectively analyzed data of 449 patients with severe COVID-19. Of these, 99 had received heparin (mainly low-molecular weight heparin) for at least 7 days. Although no difference was found in 28-day mortality of heparin users and nonusers ( $30.3 \%$ vs. $29.7 \%, p=0.910)$, the 28-day mortality of heparin users was lower than nonusers in patients with sepsis-induced coagulopathy (SIC) score $\geq 4$ ( $40.0 \%$ vs. $64.2 \%, p=0.029$ ), or D-dimer $>6$-fold of upper limit of normal ( $32.8 \%$ vs. $52.4 \%, p=0.017){ }^{21}$

Paranjpe et al analyzed the association between in-hospital anticoagulation administration and mortality among patients hospitalized with COVID-19. The researchers adjusted for age, sex, ethnicity, history of hypertension, heart failure, atrial fibrillation and type 2 diabetes; anticoagulation use prior to hospitalization; and admission date. In addition, duration of anticoagulation treatment was used as a covariate, and intubation was treated as a timedependent variable.

Of 2,773 hospitalized COVID-19 patients, 786 (28\%) received systemic anticoagulation during their hospital stay. 
The median length of stay was 5 days, while the median time from admission to anticoagulation initiation was 2 days, and the median anticoagulation treatment was 3 days. Among patients who received anticoagulation, in-hospital mortality was $22.5 \%$ with a median survival of 21 days, compared with $22.8 \%$ and a median survival of 14 days among patients who did not receive anticoagulation. ${ }^{22}$

Twenty-four patients (3\%) who received anticoagulation had bleeding events, compared with 38 patients (1.9\%) who did not receive anticoagulation. Bleeding events were more common among patients who were intubated $(7.5 \%)$ versus nonintubated patients (1.35\%). ${ }^{22}$

\section{IL-6 Pathway Blockers}

IL-6 is an important proinflammatory cytokine and in patients with COVID-19, IL-6 levels are significantly elevated and associated with adverse clinical outcomes. However, although based on sound logic, more results are necessary before either tocilizumab or sarilumab, currently used to treat rheumatoid arthritis, are considered standard of care for patients of COVID-19 with evidence of a cytokine storm. ${ }^{23}$

\section{Convalescent Plasma}

While the world awaits effective and safe vaccination against COVID-19, convalescent plasma from donors who have recovered from COVID-19 may be the most promising when used as prophylaxis or when administered shortly after symptom onset (within 14 days).

There have been three case series from China using convalescent plasma to treat COVID-19 (a total of 19 patients). Some seriously ill patients in China who received convalescent plasma therapy resulted in improved oxygenation and reduced inflammation and viral load. In this pilot study, nine patients received 1 dose of $200 \mathrm{~mL}$ convalescent plasma with neutralizing antibody titers of $>1: 640$. The median time from onset of illness to convalescent plasma transfusion was 16.5 days. Within 3 days, the patients showed improved clinical symptoms along with increase of oxyhemoglobin saturation. Lymphocyte counts increased from $0.65 \times 10^{9} / \mathrm{L}$ to $0.76 \times 10^{9} / \mathrm{mL}$ and C-reactive protein decreased from $55.98 \mathrm{mg} / \mathrm{L}$ versus $18.13 \mathrm{mg} / \mathrm{L}$. The neutralizing antibody in recipients increased in all patients and the viral load was undetectable in seven of the nine patients who had been previously afflicted with viremia. ${ }^{24}$

\section{Ventilatory Support Strategies}

The pathophysiology of COVID-19 pneumonia and respiratory failure in this disease has been a topic of discussion. It is important to understand the basic mechanism by which the virus hits humans to provide correct treatment strategies (-Table 1).

Patients with type L CARDS (COVID-19 patient with acute respiratory distress syndrome [ARDS]) have good lung compliance and can tolerate larger tidal volumes $(7-8 \mathrm{~mL} / \mathrm{kg}$ ideal body weight) than those customarily prescribed for
Table 1 Phenotypes of pneumonia ${ }^{25}$

\begin{tabular}{|c|c|c|}
\hline & L-pneumonia & $\mathrm{H}$-pneumonia \\
\hline Elastance & $\begin{array}{l}\text { Low } \\
\text { The lung } \\
\text { compliance is } \\
\text { nearly normal }\end{array}$ & $\begin{array}{l}\text { High } \\
\text { There is decrease in } \\
\text { gas volume due to } \\
\text { increased pulmonary } \\
\text { edema }\end{array}$ \\
\hline $\begin{array}{l}\text { Ventilation- } \\
\text { Perfusion ratio }\end{array}$ & $\begin{array}{l}\text { Low } \\
\text { Loss of hypoxic } \\
\text { vasoconstriction } \\
\text { results in } \\
\text { dysregulation of } \\
\text { perfusion. The } \\
\text { pulmonary artery } \\
\text { pressures are } \\
\text { normal }\end{array}$ & $\begin{array}{l}\text { High } \\
\text { Perfusion of } \\
\text { nonaerated (increase } \\
\text { in edema) depen- } \\
\text { dent region of the } \\
\text { lungs }\end{array}$ \\
\hline Lung weight & $\begin{array}{l}\text { Moderate } \\
\text { increase in lung } \\
\text { weight } \\
\text { Subpleural and } \\
\text { perifissural } \\
\text { ground glass } \\
\text { opacities seen on } \\
\text { CT scan of the } \\
\text { chest }\end{array}$ & $\begin{array}{l}\text { High } \\
\text { Determined by } \\
\text { quantitative analysis } \\
\text { on CT scan }\end{array}$ \\
\hline Recruitability & $\begin{array}{l}\text { Low } \\
\text { As the } \\
\text { nonaerated lung } \\
\text { tissue is low }\end{array}$ & $\begin{array}{l}\text { High } \\
\text { Due to increased } \\
\text { amount of } \\
\text { nonaerated lung } \\
\text { tissue }\end{array}$ \\
\hline
\end{tabular}

ARDS without worsening the risk of ventilator-induced lung injury (VILI).

Larger tidal volumes may help avoid reabsorption atelectasis and hypercapnia due to hypoventilation with lower tidal volumes. ${ }^{26}$

Ventilation perfusion mismatch, resulting from disrupted vasoregulation due to endothelial damage causing failure of hypoxic vasoconstriction in pulmonary vascular system, results in hypoxemia in the early stage of the disease. Therefore, in these early situations, increasing the fractional inspired oxygen concentration and initiating noninvasive ventilator support may improve clinical outcomes. ${ }^{26}$

High-flow nasal oxygen (HFNO) can achieve a flow rate of up to $60 \mathrm{~L} / \mathrm{min}$. HFNO reduces dead space and decreases the work of breathing and breathing frequency. It is associated with decreased mortality in hypoxemic respiratory failure. It is associated with decreased risk of progression to intubation and ICU admission.

The positive end-expiratory pressure (PEEP) should be reduced to $8-10 \mathrm{~cm} \mathrm{H2O}$, given that the recruitability is low and the risk of hemodynamic failure increases at higher levels. An early intubation may avert the transition to Type $\mathrm{H}$ phenotype.

Type $\mathrm{H}$ patients should be treated as severe ARDS, including higher PEEP, if compatible with hemodynamics, prone positioning and extracorporeal support. ${ }^{26}$ 


\section{Point-of-Care Ultrasound}

Point of care ultrasound (POCUS) has taken rapid strides in patient management in this pandemic. A simple 12-point ultrasound examination can provide a wealth of information with regard to these patients. The absence of A profile, presence of $B$ lines, and effect of changed ventilator settings on the distribution of B lines, all contribute to important feedback and early intervention, further improving clinical outcomes. ${ }^{27}$

\section{Future Perspectives}

It is pertinent to note that in about 4 months of fighting COVID-19, we already have approximately 1955 registered clinical trials and a plethora of drugs and vaccines under evaluation. COVID-19 has hit humanity hard, and as we mourn the world citizens whom we lost to this deadly disease, we also are in a race against time to find that magic bullet that will finally take down this disease.

Apart from clinical trials, it will be worth considering oral antivirals under a conditional access program for the country as was done for drug-resistant tuberculosis.

Results from WHO's SOLIDARITY trial, comprising remdesivir; lopinavir/ritonavir; lopinavir/ritonavir with interferon $\beta-1 \mathrm{a}$ and chloroquine or hydroxychloroquine shall be important to guide future therapy. ${ }^{28}$

The WHO has brought out a coordinated Global Research Roadmap in March 2020 to focus on actions that can save lives. The timeline for implementation of selected research actions aimed at identification of prioritized potential combinations by April 2020. Data on safety and efficacy of combination therapies (randomized controlled trials) is expected to be available by July 2020. Listed interventions currently being evaluated range from next generation sequencing (NGS) diagnostics in COVID-19 hosts with proposed genome analysis, $\mathrm{T}$ cell receptor repertoire and SARS-CoV-2 viral composition analysis to effectiveness of personal protection gear in long-term use..$^{29}$

Interestingly, convalescent plasma therapy, used widely in the pre antibiotic era, is being touted as a potential answer to treatment of COVID-19. ${ }^{16}$

The varied clinical manifestations of COVID-19 have brought into focus the pathophysiology of the disease and host-pathogen interactions, as study of vascular compartments and hypercoagulability during COVID-19 infection are currently being studied.

IL-1 inhibition has benefited patients with sepsis who had features of transaminitis and coagulopathy. Patients hospitalized with COVID-19 have been found to develop a hyperinflammatory syndrome in the convalescent period after defervescence of fever which can be prevented by the use of IL-1 and IL-6 inhibitors. ${ }^{16}$

It is important to note that early institution of therapy has shown better outcomes in many clinical trials. This was one of the major strategies that allowed us to gain control over the influenza pandemic. It may prove vitally important in COVID-19 as well.
Rapid progression of COVID-19 in the community has probably been through the asymptomatic group of patients. Universal masking to address the Achilles heel-asymptomatic carriers-as proposed by Gandhi et al may be the only way forward as we yearn to return to normalcy. ${ }^{30}$

As for any viral ailment, development and implementation of vaccination through the national program will be an important public health measure.

COVID-19 has challenged the whole world like never before. We hope that we defeat this disease at the earliest using our collective wisdom and efforts.

\section{Conflict of Interest}

None declared.

\section{References}

1 Jin Y, Yang H, Ji W, et al. Virology, epidemiology, pathogenesis, and control of COVID-19. Viruses 2020;12(4):E372

2 WHO. Modes of Transmission of Virus Causing COVID-19: Implications for IPC Precaution Recommendations. Available at: https://www.who.int/news-room/commentaries/detail/modesof-transmission-of-virus-causing-covid-19-implications-foripc-precaution- recommendations. Accessed May 7, 2020

3 MyGov. COVID-19 Dashboard. Available at: https://www. mygov.in/covid-19. Accessed May 7, 2020

$4 \mathrm{Bi} \mathrm{Q}, \mathrm{Wu}$ Y, Mei S, et al. Epidemiology and transmission of COVID-19 in 391 cases and 1286 of their close contacts in Shenzhen, China: a retrospective cohort study. Lancet Infect Dis 2020. Doi: 10.1016/S1473-3099(20)30287-5

5 Lauer SA, Grantz KH, Bi Q, et al. The incubation period of Coronavirus disease 2019 (COVID-19) from publicly reported confirmed cases: estimation and application. Ann Intern Med 2020;172(9):577-582

6 Kakodkar P, Kaka N, Baig MN. A comprehensive literature review on the clinical presentation, and management of the pandemic Coronavirus disease 2019 (COVID-19). Cureus 2020;12(4):e7560

7 Vetter P, Vu DL, L'Huillier AG, Schibler M, Kaiser L, Jacquerioz F. Clinical features of covid-19. BMJ 2020;369:m1470

8 MOHFW. Revised Guidelines on Clinical Management of COVID -19. Available at:https://www.mohfw.gov.in/ pdf/RevisedNationalClinicalManagementGuidelineforCO VID1931032020.pdf. Accessed June 6, 2020

9 Wang Y, Zhang D, Du G, et al. Remdesivir in adults with severe COVID-19: a randomised, double-blind, placebo-controlled, multicentre trial. Lancet 2020;395(10236):1569-1578

10 Grein J, Ohmagari N, Shin D, et al. Compassionate use of remdesivir for patients with severe Covid-19. N Engl J Med 2020;382:2327-2336

11 Business Wire. Gilead Announces results from phase 3 trial of investigational antiviral remdesivir in patients with severe COVID-19. Available at: https://www.businesswire.com/news/ home/20200429005424/en/. Accessed June 6, 2020

12 Cai Q, Yang M, Liu D, et al. Experimental treatment with favipiravir for COVID-19: an open-label control study. Engineering (Beijing) 2020. Doi: 10.1016/j.eng.2020.03.007

13 Young BE, Ong SWX, Kalimuddin S, et al; Singapore 2019 Novel Coronavirus Outbreak Research Team. Epidemiologic features and clinical course of patients infected with SARS-CoV-2 in Singapore. JAMA 2020;323(15):1488-1494

14 Cao B, Wang Y, Wen D, et al. A trial of lopinavir-ritonavir in adults hospitalized with severe Covid-19. N Engl J Med 2020;382(19):1787-1799 
15 Hung IF-N, Lung K-C, Tso EY-K, et al. Triple combination of interferon beta-1b, lopinavir-ritonavir, and ribavirin in the treatment of patients admitted to hospital with COVID-19: an open-label, randomised, phase 2 trial.Lancet 2020;395(10238): 1695-1704

16 Haematology TL; The Lancet Haematology. The resurgence of convalescent plasma therapy. Lancet Haematol 2020;7(5):e353

17 Lim J, Jeon S, Shin HY, et al. Case of the index patient who caused tertiary transmission of COVID-19 Infection in Korea: the application of lopinavir/ritonavir for the treatment of covid-19 infected pneumonia monitored by quantitative RT-PCR. J Korean Med Sci 2020;35(6):e79

18 Gautret P, Lagier JC, Parola P, et al. Hydroxychloroquine and azithromycin as a treatment of COVID - results of an open label non - randomized clinical trial Int J Antimicrob Agents 2020. Doi: 10.1016/j.ijantimicag.2020.105949

19 Caly L, Wagstaff KM, Jans DA. Nuclear trafficking of proteins from RNA viruses: potential target for antivirals? Antiviral Res 2012;95(3):202-206

20 Caly L, Druce JD, Catton MG, Jans DA, Wagstaff KM. The FDA-approved drug ivermectin inhibits the replication of SARS-CoV-2 in vitro. Antiviral Res 2020;178:104787

21 Tang N, Bai H, Chen X, Gong J, Li D, Sun Z. Anticoagulant treatment is associated with decreased mortality in severe coronavirus disease 2019 patients with coagulopathy. J Thromb Haemost 2020;18(5):1094-1099

22 Paranjpe I, Fuster V, Lala A, et al. Association of treatment dose anticoagulation with in-hospital survival among hospitalized patients with COVID-19. J Am Coll Cardiol 2020. Doi: 10.1016/j. jacc.2020.05.001
23 Coomes EA, Haghbayan $H$, Interleukin-6 in COVID-19: a systematic review and meta- analysis medRxiv2020; Doi: $10.1101 / 2020.03 .30 .20048058$

24 Brown BL, McCullough J. Treatment for emerging viruses: convalescent plasma and COVID-19. Transfus Apheresis Sci 2020;59(3):102790

25 Gattinoni L, Chiumello D, Caironi P, et al. COVID-19 pneumonia: different respiratory treatments for different phenotypes? Intensive Care Med 2020. Doi: 10.1007/s00134-020-06033-2

26 Marini JJ, Gattinoni L. Management of COVID-19 respiratory distress. JAMA 2020. Doi: 10.1001/jama.2020.6825

27 Smith MJ, Hayward SA, Innes SM, Miller ASC, Point-of-care lung ultrasound in patients with COVID-19 - a narrative review Anaesthesia 2020. Doi: 10.1111/anae.15082

28 World Health Organisation. "Solidarity" Clinical Trial for COVID-19 Treatments. Available at: https://www.who.int/ emergencies/diseases/novel-coronavirus-2019/globalresearch-on-novel-coronavirus-2019-ncov/solidarity-clinical-trial-for-covid-19- treatments. Published 2020. Accessed May 9, 2020

29 World Health Organisation. 2019 Novel Coronavirus: A Coordinated Global Research Roadmap to Respond to TheD-19 Epidemic. Available at: https://www.who.int/blueprint/priority-diseases/key-action/Roadmap-version- FINAL-for-WEB. pdf?ua=1. Accessed June 6, 2020

30 Gandhi M, Yokoe DS, Havlir DV, Asymptomatic transmission, the Achilles' heel of current strategies to control Covid-19. N Engl J Med 2020. Doi: 10.1056/NEJMe2009758 